\title{
Diagnostic Value of Transthoracic Ultrasonography for COVID-19 Pneumonia: A Cross-Sectional Study
}

\author{
Abdul Rasheed Qureshi ${ }^{1,2}$, Huma Bilal ${ }^{3}$, Muhammad Sajid ${ }^{3}$, Zeeshan Ashraf ${ }^{4}$ \\ ${ }^{1}$ Head, Department of Pulmonology, Gulab Devi Teaching Hospital Lahore, Pakistan \\ 2 Director, Institute of Biotechnology, Gulab Devi Educational Complex Lahore, Pakistan \\ ${ }^{3}$ Medical Officer, Gulab Devi Teaching Hospital Lahore, Pakistan \\ ${ }^{4}$ Lecturer, Department of Statistics, Gulab Devi Educational Complex Lahore, Pakistan
}

\begin{abstract}
AB S TR ACT
Background: Corona virus infection requires early detection and precise diagnosis for an effective control in community. COVID-19 PCR is the gold standard diagnostic test having low sensitivity and adequate availability constraints. Because of high false negative rate, a large number of undetected patients remain a constant source of inadvertent disease dissemination in the community. This study was conducted to determine the diagnostic usefulness of transthoracic ultrasonography for the detection of COVID-19 pneumonia during the ongoing pandemic.

Material and Methods: This cross-sectional study was conducted in the Pulmonology OPD of Gulab Devi Teaching Hospital, Lahore-Pakistan from April 1, 2020 to July 30, 2020. A total of 237 patients with dry cough, fever and shortness of breath of sudden onset, clinically diagnosed as COVID-19 pulmonary infection, were included in the study. Patients were investigated with chest $\mathrm{x}$-ray/HRCT, transthoracic ultrasonography, COVID-19 RT-PCR on nasopharyngeal samples and hematological tests. Sensitivity, Specificity, PPV (positive predictive value), NPV (negative predictive value) and diagnostic accuracy of transthoracic ultrasonography was calculated with $P$-value $<0.05$ considered as statistically significant. SPSS-26 software was used for statistical analysis.

Results: Of the total 237 patients (mean age $41.23 \pm 17.5$ years) presenting with complaints of a pulmonary infection, 228 were diagnosed as COVID-19 pulmonary infection by transthoracic ultrasonography and PCR. Bilateral lesions and B-lines were the most frequent characteristic features on ultrasonography ( $n=237$ patients; 100\%). Transthoracic ultrasound diagnosed 228 patients as cases of COVID-19 pneumonia with $100 \%$ sensitivity, $66.67 \%$ specificity, $98.7 \%$ PPV, $100 \%$ NPV, and 98.73\% diagnostic accuracy. Only 135/228 (59.21\%) patients were diagnosed by RT-PCR. The $P$ value by Fisher exact test was highly significant at 0.001 (cut-off $P$-value $<0.05$ ).

Conclusions: Transthoracic ultrasonography is a useful diagnostic tool with a high sensitivity, is free from radiation exposure and capable of providing foundations for evidence based, early detection of coronavirus pneumonia in a pandemic situation.

Key words: Coronavirus pneumonia, Diagnostic accuracy, Pandemic, Transthoracic ultrasonography

Authors' Contribution:

${ }^{1}$ Conception; Literature research;

Correspondence:

Abdul Rasheed Qureshi

manuscript design and drafting; 2,3 Critical

Email:drrasheed57@gmail.com

Article info:

Received: June 29, 2020

Accepted: September 15, 2020

analysis and manuscript review; Dato

analysis; Manuscript Editing.
\end{abstract}

Cite this article. Qureshi AR, Bilal H, Sajid M, Ashraf Z. Diagnostic value of Transthoracic Ultrasonography for COVID-19 Pneumonia: A Prospective Study. J Islamabad Med Dental Coll. 2020; 9(3): 158-167. Doi: 10.35787/jimdc.v9i3.581
Funding Source: Nil

Conflict of Interest: Nil 


\section{Introduction}

The outbreak of unusual viral pneumonia started in China, in December 2019 and was later on recognized as coronavirus disease-2019 (COVID19). ${ }^{1}$ By the end of July 2020 , this fatal disorder had infected about $170,53,642$ patients globally with a death toll of 6,66,461 people, worldwide. COVID-19 disease was subsequently declared as a Public Health Emergency of International Concern (PHEIC) by WHO (World Health Organization). ${ }^{2}$ This disease is highly contagious and number of cases multiplied rapidly over the globe. According to the current literature, one infected patient may lead to more than two new patients and at times the disease is too drastic to be controlled and the outcome is disastrous. Coronavirus infection has affected not only the general public but senior health care providers and eminent figures from the society have been lost to this lethal disease. At the end of July, there were 279,146 confirmed cases and 5,970 mortalities in Pakistan, indicating the load and damage caused by this devastating sickness.

Presentation of COVID-19 disease ranges from mild to extensive, requiring mechanical ventilation. Early diagnosis is essential for effective control to preclude further dissemination. COVID-19 RT-PCR (Reverse Transcription Polymerase Chain Reaction) is the gold standard diagnostic test, which has high specificity but low sensitivity of around 60\%, meaning that about $40 \%$ cases with disease are missed. ${ }^{3}$ Furthermore, this test is not capable of calculating the magnitude of the disease and the expected decline in respiratory functions. Chest $X-$ ray PA view can identify moderate to severe disease but is unable to detect early involvement because less than $3.0 \mathrm{~mm}$ nodule is not resolved by CXR (chest $x$-ray). In this way, a large number of patients are not diagnosed and remain a constant source of inadvertent dissemination within the community.
Many researchers have been recommending theuse of HRCT (High-Resolution Computed Tomography) chest as a screening tool for COVID-19 pneumonia in the setting of an epidemic. ${ }^{4} \mathrm{HRCT}$ - chest Can provide immediate results with a high sensitivity and adequate specificity for COVID-19 pneumonia, but it is neither cost-effective nor feasible and safe for use in pregnant patients and children due to hazards of ionizing radiations. ${ }^{5}$ Similarly, due to same reasons, CXR with limited sensitivity and specificity, cannot be considered as an alternative tool for detecting COVID-19 pneumonia.

The ultrasound, using sound waves rather than ionizing radiations, with no deleterious effect on young children and pregnant patients, can be used safely without any fear of mutagenic effects. Recently, researchers have indicated the usefulness of transthoracic ultrasound for detecting COVID-19 pneumonia. Particularly, Chinese and Italian task forces have recommended that pulmonary ultrasound can be a useful tool for diagnosing and monitoring the course of COVID-19 pneumonia. ${ }^{6,7}$

Chest ultrasonography is under-used in Pakistan due to lack of adequate understanding and training in the field. Although, it is being adequately used for the detection and aspiration of pleural fluid but its use in detecting pulmonary parenchymal lesions is still in the beginning phase. According to research reports on HRCT, COVID-19 pneumonic lesions are distributed in peripheral part of the lung, which can be easily recognized by chest ultrasound. ${ }^{8}$ It has also been reported that transthoracic ultrasonography is superior to CXR and HRCT for the investigation of pneumonia with an added advantage of use at point of care, easy availability, cost-effectiveness, repeatability and absence of radiation exposure. ${ }^{9}$ 
B-lines are generated by interaction of ultrasound waves with sub-pleural consolidation or irregular pleural margins, indicating COVID-19 pulmonary disease, in the setting of an epidemic. But these lines can also be seen in other interstitial diseases of various etiologies. ${ }^{10-12}$ The Chinese authors named this sign as "waterfall", without further elaborating it. ${ }^{13}$ The use of bedside ultrasound could reduce the need of CXR and HRCT and shows amazing results during the follow-up of pneumonia.

The current study was conducted to determine the diagnostic usefulness of transthoracic ultrasonography for the detection of COVID-19 pneumonia, during the ongoing pandemic.

\section{Material and Methods}

This cross-sectional study was conducted in the Pulmonology Department of Gulab Devi Teaching Hospital, Lahore Pakistan (a 1500-beded tertiary care hospital in the heart of Lahore, the capital city of Punjab) from A pril 1, 2020 to July 30, 2020. After obtaining informed consent, 237 patients with oneweek history of dry cough, fever and shortness of breath (of sudden onset) and a presumptive diagnosis of COVID-19 pulmonary infection were included in the study. Patients with bronchial asthma, tuberculosis, bronchiectasis, COPD, ILD, and overt heart failure were excluded. Sample size was calculated with the help of online Open-Epi calculator for cross-sectional studies. A sample size of 234 was calculated against the prevalence of $16.3 \%{ }^{14}$ with a confidence interval of $95 \%$. But we included 237 patients to compensate for the missing data. Ethical Approval was obtained from Institutional Review Board of the hospital (vide: No. Admin/GDEC/20/326).

The patients were subjected to X-ray chest PA view and transthoracic ultrasound. All patients underwent routine hematological tests including white blood cell count, lymphocyte count, C-reactive protein, serum $\mathrm{LDH}$, Ferritin and D-Dimer test in pertinent cases. Nasopharyngeal samples from patients were tested with RT-PCR for COVID-19. Xray and HRCT chest images were evaluated. Transthoracic ultrasonography was performed by qualified and experienced radiologist using Sonovista FX (Siemens) and Toshiba-Japan machines with convex and linear probes, utilizing 3-12-MHZ frequency. Guidelines of World Federation for Ultrasound in Medicine and Biology (WFUMB) for performing a safe ultrasonographic examination and ensuring use of clean equipment in the COVID-19 scenario were strictly followed during the study. ${ }^{15}$

Patients were scanned in supine and sitting positions. Anterior, lateral and posterolateral scanning was performed. Anterior scanning was done between parasternal and anterior axillary lines. Area for lateral scanning was located between anterior and posterior axillary lines while posterolateral scanning was done beyond the posterior axillary line, in subscapular and interscapular regions. All regions were scanned in upper and lower parts. Following sonographic signs were noted: Any scan with regular sliding, A-lines all over the chest and absent $B$-lines, was considered as normal lung scan. A COVID-19 scan included ${ }^{11,13,16}$ (Figure-1): 1. Bilateral patchy involvement; 2 . B-lines which are vertical lines, originating from pleural surface, running down to the lower edge of the screen without fading away. These lines can be single, multiple, discrete or confluent (Figure-2); 3 . Bilateral patchy $\mathrm{B}$-lines, appearing and disappearing during inspiration and expiration, looking like on-off phenomenon of a light beam; 4. A cluster of B-lines creating the appearance of white-lung (Figure-2); 5 . Irregular or fragmented pleural line with preserved sliding sign (Figures 1 \& 2); 6. Sub-pleural patchy consolidations of variable sizes with a light beam and irregular pleural line; 7. Pleural Effusion-uncommon sign. 
CXR, HRCT, ultrasound-findings, RT-PCR reports, demographic and clinical data of 237 patients were recorded on a proforma. Clinical diagnosis was established by correlating clinicopathological data and diagnostic accuracy of COVID-19-PCR and transthoracic ultrasound was calculated by considering clinical diagnosis as reference. Sensitivity, Specificity, PPV (positive predictive value), NPV (negative predictive value), diagnostic accuracy and $P$-value were calculated for both modalities.

SPSS-26 software was used for statistical analysis. Quantitative data was represented by means and standard deviations and categorical variables were expressed by frequency (percentage). Fisher exact test was used for comparison and a $P$-value $<0.05$ was considered as significant.

\section{Results}

This study included 237 patients with a mean age of 41.23 years \pm 17.5 (age range 18 to 87 years). About 126 cases (53.16\%) were above the age of 50 years. Of these, 144 patients were male while 93 were female with a male to female ratio of 1.54 . All patients showed positive history of contact from their familial clusters. Patients presented with typical respiratory complaints as shown in Table I. History of cigarette smoking was found in 67 (28.27\%) patients, diabetes mellitus in 77 (32.48\%) and obesity was seen in 17 cases $(7.17 \%)$, respectively. About 163 patients (68.77\%) displayed elevated CRP levels with mean CRP level of $9.6 \pm 6.8$ $\mathrm{mg} / \mathrm{dl}$. In only 135/237 (56.96\%) patients, COVID-19, PCR-Test was positive.

Of the 237 clinically diagnosed cases, 231 were diagnosed COVID-19 pneumonia by transthoracic ultrasonography while 06 reports were equivocal. All cases displayed bilateral radiological abnormalities (Table I). The B-line morphology revealed 111 (46.83\%) separate B-lines, 103 (43.46\%) confluent and 23 (9.7\%) compact B-lines, respectively. Anterior scan was positive in 64 patients (27\%) while 173 cases (73\%) exhibited abnormal findings in lateral and posterior scans. Maximum findings were found in posterior scans in inter-scapular and subscapular regions. Detailed radiological morphologies and their frequencies were recorded (Table I). Regarding disease severity, $64.97 \%$ patients had mild disease, $31.22 \%$ moderate while only $3.79 \%$ had severe disease.

\begin{tabular}{|l|c|}
\hline \multicolumn{2}{|c|}{$\begin{array}{c}\text { Table I: Frequency distribution of clinical and } \\
\text { ultrasound features on Transthoracic } \\
\text { ultrasonography in COVID-19 Patients (n=237) }\end{array}$} \\
\hline Clinical features & $\mathbf{n}(\%)$ \\
\hline High grade fever & $189(79.74)$ \\
\hline Dry cough & $137(57.80)$ \\
\hline Shortness of breath & $209(88.18)$ \\
\hline Muscle pain and weakness & $170(71.72)$ \\
\hline Chest pain & $71(29.95)$ \\
\hline Headache & $46(19.40)$ \\
\hline Diarrhea & $27(11.39)$ \\
\hline Ultrasound Features & $\mathbf{n}(\%)$ \\
\hline Bilateral lesions & $237(100)$ \\
\hline B-lines & $237(100)$ \\
\hline Pleural surface irregularities & $206(86.91)$ \\
\hline Sub-pleural consolidation & $183(77.21)$ \\
\hline Pleural effusion & $01(0.42)$ \\
\hline Pericardial effusion & 01 \\
\hline Pneumothorax & $01(0.42)$ \\
\hline
\end{tabular}

About 228 cases were diagnosed as COVID-19 pulmonary infection on clinical grounds by correlating ultrasound findings, history, physical examination, PCR and blood reports. RT-PCR performed on nasopharyngeal samples detected COVID-19 in 135 patients (135/228; 59.21\%). Bilateral shadows were noted in 126 chest $x$-rays while 111 reports were unremarkable. Efficacy of RT-PCR was calculated by considering the clinical diagnosis as reference and 135 TP (true-positive) cases, 00 (zero) FP (false positive) cases, $93 \mathrm{FN}$ (false negative) cases and 09 TN (true negative) cases (Table II). Similarly, efficacy of transthoracic ultrasound was determined by considering TP: 228, FP 03, FN: 00 and TN cases: 06 (Table II). 

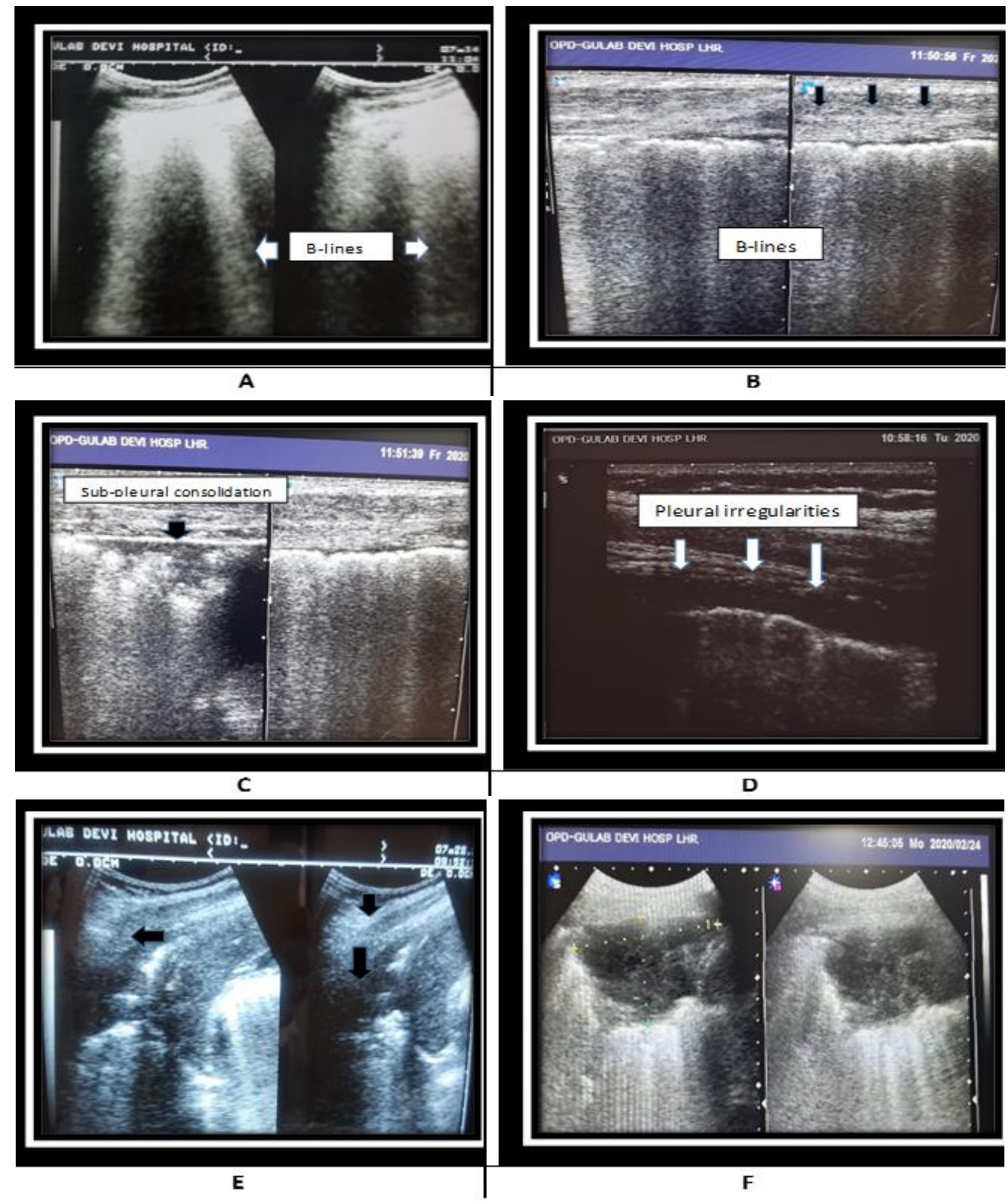

Figure 1: Ultrasound morphology of COVID-19 pulmonary infection. A: B-lines, B: Irregular pleural line, B-lines, bilateral haze, C: Sub-pleural consolidation, irregular pleura and B-lines. D: Irregular pleura and B-lines. E: Consolidation with air-bronchogram. F: Consolidation without air-bronchogram. 


\begin{tabular}{|l|c|c|}
\hline \multicolumn{3}{|c|}{ Table II: Diagnostic accuracy of PCR and Ultrasound for COVID-19 Pneumonia (n=237) } \\
\hline \multicolumn{3}{|c|}{ Efficacy of RT-PCR } \\
\hline Statistics & Value (\%) & 95\% Confidence interval \\
\hline Sensitivity & 59.21 & 52.53 to 65.65 \\
\hline Specificity & 100 & 66.37 to 100 \\
\hline PPV & 100 & 7.64 to 10.17 \\
\hline NPV & 8.82 & 54.23 to 67.02 \\
\hline Diagnostic Accuracy & 60.76 & 98.40 to 100 \\
\hline \multicolumn{3}{|c|}{} \\
\hline Sensitivity & 100 & 29.93 to 92.51 \\
\hline Specificity & 66.67 & 96.79 to 99.48 \\
\hline PPV & 98.70 & \\
\hline NPV & 100 & 96.35 to 99.74 \\
\hline Diagnostic Accuracy & 98.73 & \\
\hline
\end{tabular}

PPV-Positive predictive value; NPV-Negative predictive value

The P-value by Fisher exact test was highly significant at .001 (cut-off $\mathrm{P}$ - value $<0.05$ ).

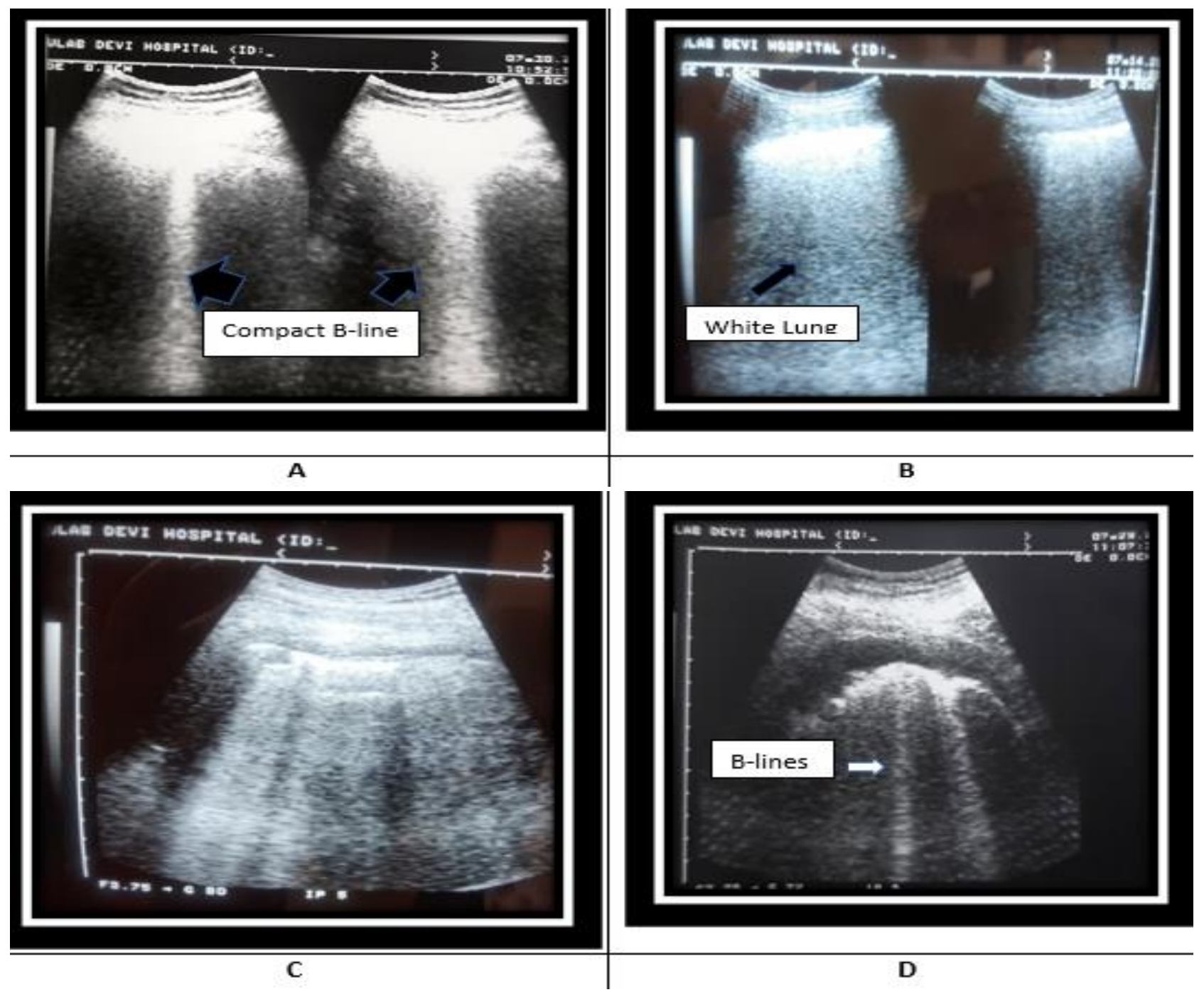

Figure 2: B-line morphology in COVID-19 pneumonia. A: Compact B-line. B: White lung sign. C: Multiple confluent Blines. D: Multiple discrete B-lines. 


\section{Discussion}

In the background of low detection rate of COVID-19 pulmonary disease by RT-PCR, this study highlights the usefulness of transthoracic ultrasonography with superior efficacy which can be a benchmark for evidence-based management and good disease control in the community.

The age of the study population ( $n=237$ patients) ranged between 18 to 87 years. Mean age was 41.23 years ( \pm 17.52$)$, which is comparable to the report of Hu and co-authors (mean age 39.2 years \pm 9.6 ) from Wuhan, China. ${ }^{17}$ The male gender preponderance of this study is in agreement with the publishedstudies of Lomoro et al. and Bhandari et al..$^{18,19}$ The reduced susceptibility of females to COVID-19infection could be due to the protection from $\mathrm{X}$-chromosome and sex hormones, providing innate and adaptive immunity. ${ }^{20}$

In this study, 126 patients (53.16\%) were above the age of 50 years, indicating that this age can be a relative risk factor for COVID-19 pulmonary disease. Similarly, co-morbidities like obesity, cigarette smoking and diabetes mellitus, collectively accounting for 161 cases, may have a positive association with the respiratory disease, which is well supported by the literature. ${ }^{21,22}$

About $64.97 \%$ patients had mild disease while less than $4 \%$ cases had severe COVID-19 pneumonia. This finding may be due to weak virulence of the virus, and may be a cause for a fatality rate of less than $3 \%$ in Asia as compared to European countries and the USA where case fatality rate ranged between $10-15 \% .^{23}$

RT-PCR is regarded as the gold standard diagnostic test but has low sensitivity of around $60 \%$, due to which it is unable to detect $40 \%$ patients with disease. These patients feel satisfied with the negative report, attribute their symptoms to minor ailments, do not seek any medical help for the fatal disorder and remains a constant source of inadvertent disease dissemination in the community and non-COVID-19 wards. These patients may enter silently into severe COVID-19 pulmonary disease, requiring mechanical ventilation. In this study, only 135 patients (59.21\%) were diagnosed by PCR while 93 cases $(40.79 \%)$ were missed.

Chest $\mathrm{x}$-ray detected disease in $53.16 \%$ cases while $46.83 \%$ were equivocal but abnormal findings were noted on HRCT of the same patients. This finding is in agreement with the report of Jacobi and coauthors. ${ }^{24}$ The low sensitivity of CXR may be, because, majority of our cases belonged to mild category with subtle disease which could not be manifested on CXR. ${ }^{25}$ The efficacy of HRCT is superior to those of CXR and PCR (around 90\%) but the issues of cost, unavailability and exposure to ionizing radiations are main constraints.

As this virus is highly contagious and very unpredictable in behavior, its control needs early diagnosis for timely isolation or quarantine. Not every COVID-19 patient requires mechanical ventilation on the first day of infection. It takes ample time for developing mild to severe COVID-19 disease. The track of transition from mild to severe disease can be blocked by early diagnosis and prompt management. In these circumstances, there is an urgent need of some alternative high sensitivity test, capable of detecting the disease early and with precision.

On the other hand, lung ultrasound has shown superior sensitivity for acute respiratory distress syndrome. ${ }^{26}$ Because of peculiar ultrasound features, it can successfully be used for early approach to COVID-19-suspects. In Italy, lung ultrasound has been proposed as an alternative to HRCT for patients suspected of having COVID-19. ${ }^{2728}$ Although, ultrasound signs for covod-19 are not as specific as RT-PCR, but correlation with history and 
blood tests in the milieu of a pandemic provide adequate specificity. B-lines, the earliest manifestation, correspond to ground glass appearance on HRCT can be of tremendous help for timely isolation and triage of the suspects. ${ }^{29}$

In this study, ultrasound patterns like pleural irregularities, sub-pleural consolidation and vertical artifacts (B-lines), discrete, confluent and white lung, pointing towards ARDS were encountered. With further disease progression, consolidation with air-bronchogram sign was exhibited. By serial scanning, progression or resolution of the disease can be assessed successfully. Only one case of pleural effusion and pneumothorax each, was noted. Pleural effusion was bilateral and cardiogenic, secondary to coronary bypass surgery, superimposed by COVID-19. Pleural effusion is not common in COVID-19 but Huang et al. reported that it may be noted in severe COVID-19 pneumonia. ${ }^{30}$ More than 50\% pneumothorax was found on the left side in a 72-year-old male patient of severe pulmonary COVID-19 with diabetes mellitus, which is a rare complication. When identified, there is always evidence of positive pressure ventilation, but our patient developed pneumothorax on the day third of the fever, without any ventilation. Probability of pneumothorax is due to advanced alveolar damage, bronchiolar distortion and narrowing resulting in bullae formation while dry irritating cough, increases the intrapulmonary pressure, precipitating bullae rupture and pneumothorax.

Literature search showed that the sensitivity of PCR is as low as $60 \%$, which can be enhanced utilizing tracheal aspirates or BAL (broncho-alveolar lavage) but there is a very high risk of aerosolization and disease transmission to healthcare workers during these procedures. ${ }^{31,32}$ Transthoracic ultrasound is free of such drawbacks and capable of providing rapid and valuable information for triaging isolation and treatment procedures.
The current study displayed the efficacy of Transthoracic ultrasound with $100 \%$ sensitivity, $66.67 \%$ specificity and $98.73 \%$ diagnostic accuracy for pulmonary COVID-19 disease. The comparative efficacy revealed low sensitivity of RT-PCR. There is a statistically significant difference between the two modalities and the results show that ultrasound has remarkable capability of detecting COVID-19 pulmonary disease during an epidemic.

Thoracic Ultrasonography has numerous advantages over CXR and HRCT like, being a bed-side procedure, widely available, inexpensive, easily performed, no involvement of ionizing radiation or contrast medium and widely accepted by the patient. It can be tremendously useful in scenarios where HRCT is not available or undesirable, especially in pregnancy and immobile patients.

In summary, B-lines, consolidation and pleural line abnormalities are common abnormal findings in COVID-19 pneumonia. Bilateral involvement is frequent, maximum findings are found in posterior scan in inter-scapular and sub-scapular regions. That is why lung ultrasound could serve as a safe and valuable tool for the detection and follow-up of COVID-19 disease.

We have no hesitation in suggesting that chest ultrasound can be used during an epidemic, especially where high sensitivity, reliable laboratory testing tool for COVID-19 infection is not available. We hope, this study findings can facilitate early identification and good management of symptomatic and suspected COVID-19 pneumonia cases by judicious use of ultrasound thorax, under strict biosafety measures.

Limitation: The main limitation of our study is that it is a single center study performed on a limited sample size, hence the study may not reflect the full range of sonographic morphology and distribution patterns of COVID-19 pulmonary disease. To further elucidate, a multi-center study with a larger sample 
size can additionally explore the sonographic behavior of this disease.

\section{Conclusion}

Transthoracic ultrasonography, having high sensitivity for COVID-19 pulmonary infection, is an excellent modality with a positive role in early detection and management of pulmonary coronavirus disease during pandemic. It precludes radiation hazards and is of tremendous help as point-of-care evaluation tool in immobile patients.

\section{Acknowledgment}

The authors are thankful to Dr. Shahid Raza and Muhammad Tahir for their valuable co-operation during the study.

\section{References}

1. Chen N, Zhou M, Dong X, Qu JM, Gong FY, Han Y, et al. Epidemiological and clinical characteristics of 99 cases of 2019 novel coronavirus pneumonia in Wuhan, China: a descriptive study. Lancet. 2020; 395(10223): 507-513. Doi: 10.1016/S0140-6736(20)30211-7

2. World Health Organization, 2020. Novel Coronavirus (2019-nCoV) Situation Report-1. Available at: https://www.who.int/emergencies /diseases/novelcoronavirus-2019/situation-reports. Accessed June 5, 2020.

3. Kanne JP. Chest CT findings in $\mathbf{2 0 1 9}$ novel coronavirus (2019-nCoV) infections from Wuhan, China: key points for the radiologist. Radiol. 2020; 295(1): 200241. Doi:10.1148/radiol.2020200241

4. Ai T, Yang Z, Hou H, Zhan C, Chen C, Lv W, et al. Correlation of chest CT and RT-PCR testing in coronavirus disease 2019 (COVID-19) in China: A report of 1014 cases. Radiol. 2020; 296(2): E32-E40.

5. Moro F, Buonsenso D, Moruzzi MC, Inchingolo R, Smargiassi A, Demi $L$, et al. How to perform lung ultrasound in pregnant women with suspected COVID19 infection. Ultrasound Obstet Gynecol. 2020; 55(5): 593-598 Doi: 10.1002/uog.22028

6. Peng QY, Wang $X T$, Zhang LN. Chinese Critical Care Ultrasound Study Group (CCUSG). Findings of lung ultrasonography of novel corona virus pneumonia during the 2019-2020 epidemic. Intensive Care Med 2020. 46(5): 849-850. Doi: 10.1007/s00134-02005996-6.

7. Soldati G, Smargiassi A, Inchingolo R, Buonsenso D, Perrone T, Briganti DF, et al. Is there a role for lung ultrasound during the COVID-19 pandemic? J Ultrasound Med 2020; 39(7): 1459-1462. Doi: 10.1002/jum.15284

8. Han X, Jiang N, Cao Y, Alwalid O, Gu J, Fan Y, et al. Radiological findings from 81 patients with COVID-19 pneumonia in Wuhan, China: a descriptive study. Lancet Infect Dis. 2020; 20(4): 425-34. Doi:10.1016/S1473-3099(20)30086-4

9. Shah VP, Tunik MG, Tsung JW. Prospective evaluation of point-of care ultrasonography for the diagnosis of pneumonia in children and young adults. JAMA Pediatr. 2013;167(2):119-25.

10. Volpicelli G, Lamorte A, Villén T. What's new in lung ultrasound during the COVID-19 pandemic. Intensive Care Med. 2020; 46: 1445-1448. Doi: 10.1007/s00134-020-06048-9

11. Volpicelli G, Gargani L. Sonographic signs and patterns of COVID-19 pneumonia. Ultrasound J. 2020; 12: 22. Doi: 10.1186/s13089-020-00171-w

12. Reissig A, Copetti R, Mathis G, Mempel C, Schuler A, Zechner $\mathrm{P}$, et al. Lung ultrasound in the diagnosis and follow-up of community-acquired pneumonia: a prospective, multicenter, diagnostic accuracy study. Chest. 2012; 142(4): 965-72. Doi: 10.1378/chest.120364

13. Tan G, Lian X, Zhu Z, Wang Z, Huang F, Zhang Y, et al. Use of Lung Ultrasound to Differentiate Coronavirus Disease 2019 (COVID-19) Pneumonia from Community-Acquired Pneumonia. Ultrasound Med Biol. 2020; 46(10): 2651-2658. Doi: 10.1016/j.ultrasmedbio.2020. 05.006.

14. Accuracy of Lung Ultrasound in the Diagnosis of covid19 Pneumonia (POCUSars-CoV-2). https://clinicaltrials.gov/ct2/show/NCT04370275

15. Abramowicz JS, Basseal JM. World federation for ultrasound in medicine and biology position statement: how to perform a safe ultrasound examination and clean equipment in the context of COVID-19. Ultrasound Med Biol. 2020; 46(7): 1821-6. Doi: 10.1016/j.ultrasmedbio.2020.03.033 
16. Huang $Y$, Wang $S$, Liu $Y$, Zhang $Y$, Zheng $C$, Zheng $Y$, et al. A Preliminary Study on the Ultrasonic Manifestations of Peripulmonary Lesions of NonCritical Novel Coronavirus Pneumonia (COVID-19) (February 26, 2020). Doi: 10.2139/ssrn.3544750. Available at SSRN: https://ssrn.com/ abstract $=3544750$

17. Hu Q, Guan H, Sun Z, Huang L, Chen C, Ai T, et al. Early CT features and temporal lung changes in COVID-19 pneumonia in Wuhan, China. Eur J Radiol. 2020; 128: 109017. Doi:10.1016/j.ejrad. 2020.109017

18. Lomoro P, Verde F, Zerboni F, Simonetti L, Borghi C, Fachinetti C, et al. COVID-19 pneumonia manifestations at the admission on chest ultrasound, radiographs, and CT: single-center study and comprehensive radiologic literature review. Eur J Radiol Open. 2020; 7: 100231. Doi:10.1016/j. ejro.2020.100231

19. Bhandari S, Singh A, Bagarhatta M, Rankawat G, Dube A, Kakkar S, et al. Evaluation of clinicoRadiological profile and correlation with ultrasonography of the chest in coronavirus disease 2019 pneumonia. Indian J Med Spec 2020; 11(2): 705. Doi: 10.4103/INJMS.INJMS_55_20

20. Jaillon S, Berthenet K, Garlanda C. Sexual dimorphism in innate immunity. Clin Rev Allergy Immunol. 2019; 56(3): 308-321. Doi: 10.1007/ s12016-017-8648-x

21. Wang XF, Shi GC, Wan HY. Clinical features of three avian influenza H7N9 virus-infected patients in Shanghai. Clin Respir J. 2014; 8(4): 410-416. Doi: 10.1111/crj.12087

22. Kornum JB, Thomsen RW, Riis A, Lervang $H H$, Schønheyder HC, Sørensen HT. Diabetes, glycemic control, and risk of hospitalization with pneumonia: a population-based case-control study. Diabetes Care. 2008; 31(8): 1541-1545. Doi:10.2337/dc080138

23. Salahuddin N. The COVID-19 pandemic. JPMA. 2020; 70(5), S4-6. Doi: 10.5455/JPMA.02
24. Jacobi A, Chung $M$, Bernheim A, Eber C. Portable chest X-ray in coronavirus disease-19 (COVID-19): A pictorial review. Clin Imaging. 2020; 64: 35-42. Doi: 10.1016/j.clinimag.2020.04.001

25. Aljondi R, Alghamdi S. Diagnostic Value of Imaging Modalities for COVID-19: Scoping Review. J Med Internet Res. 2020; 22(8): e19673. Doi: 10.2196/19673

26. Peng $Q$, Wang $X$, Zhang L. Findings of lung ultrasonography of novel corona virus pneumonia during the 2019-2020 epidemic. Intensive Care Med. 2020; 46(5): 849-850 Doi: 10.1007/s00134020-05996-6

27. Lichtenstein DA, Malbrain MLNG. Lung ultrasound in the critically ill (LUCI): A translational discipline. Anaesthesiol Intensive Ther. 2017; 49(5): 430-436. Doi:10.5603/AIT. a2017.0063

28. Poggiali E, Dacrema A, Bastoni D, Tinelli V, Demichele E, Ramos PM, et al. Can lung US help critical care clinicians in the early diagnosis of novel coronavirus (COVID-19) pneumonia? Radiology. 2020; 295(3): E6. Doi: 10.1148/radiol. 2020200847

29. Soldati G, Smargiassi A, Inchingolo R, Buonsenso D, Perrone $T$, Briganti DF, et al. Is there a role for lung ultrasound during the COVID-19 pandemic? J Ultrasound Med. 2020; 39(7): 1459-62. Doi: 10.1002/jum.15284

30. Huang C, Wang Y, Li X, Ren L, Zhao J, Hu Y, et al. Clinical features of patients infected with 2019 novel coronavirus in Wuhan, China. Lancet. 2020; 395(10223): 497-506 Doi: 10.1016/S01406736(20)30183-5.

31. Fang $Y$, Zhang $H$, Xie J, Lin $M$, Ying $L$, Pang $P$, et al. Sensitivity of Chest CT for COVID-19: Comparison to RT-PCR. Radiology 2020; 296(2): E115-E117. Doi: 10.1148/radiol.2020200432

32. Wölfel R, Corman VM, Guggemos W, Seilmaier M, Zange S, Müller MA et al. Virological assessment of hospitalized patients with COVID-2019. Nature. 2020; 581(7809): 465-469. Doi:10.1038/s41586020-2196-x 